\title{
Instability of nonminimally coupled scalar fields in the spacetime of slowly rotating compact objects
}

\author{
Raissa F. P. Mendes, ${ }^{1, *}$ George E. A. Matsas, ${ }^{1, \dagger}$ and Daniel A. T. Vanzella ${ }^{2, \$}$ \\ ${ }^{1}$ Instituto de Física Teórica, Universidade Estadual Paulista, Rua Dr. Bento Teobaldo Ferraz 271, \\ 01140-070 São Paulo, São Paulo, Brazil \\ ${ }^{2}$ Instituto de Física de São Carlos, Universidade de São Paulo, \\ Caixa Postal 369, 13560-970 São Carlos, São Paulo, Brazil
}

(Received 21 July 2014; published 18 August 2014)

\begin{abstract}
Nonminimally coupled free scalar fields may be unstable in the spacetime of compact objects. Such instability can be triggered by classical seeds or, more simply, by quantum fluctuations giving rise to the so-called "vacuum awakening effect." Here, we investigate how the parameter space that characterizes the instability is affected when the object gains some rotation. For this purpose, we focus on the stability analysis of nonminimally coupled scalar fields in the spacetime of slowly spinning matter shells.
\end{abstract}

DOI: $10.1103 /$ PhysRevD.90.044053

PACS numbers: 04.62.+v, 04.40.Dg, 95.30.Sf

\section{INTRODUCTION}

In Refs. [1,2] it was found that quantum fluctuations of certain nonminimally coupled free scalar fields defined in the spacetime of some relativistic stars can undergo an exponential amplification in time (see also Ref. [3] for a comprehensive discussion). This "vacuum awakening effect" can be seen as the quantum counterpart of the classical linear instability experienced by these nonminimally coupled fields in such spacetimes [4], or, more generally, of the classical instability observed in certain scalar-tensor theories $[5,6]$.

A particularly interesting implication of this instability (neglecting restabilization mechanisms [7]) is the possibility of ruling out certain classes of nonminimally coupled scalar fields by, e.g., determining the mass-to-radius ratio of relativistic stars with known equations of state. For this purpose, it is interesting to allow for natural deviations of the symmetry assumptions imposed on the stellar models considered in Ref. [2], such as spherical symmetry and staticity, and investigate whether the conclusions would change significantly. This was partially done in Ref. [8], where a class of static spheroidal shells was taken as the source of the gravitational field, and it was shown how the space of parameters that trigger the instability changes when increasingly higher deviations from spherical symmetry are considered. The aim of the present paper is to complement that analysis by studying the effects of rotation, which is an ubiquitous and often important property of astrophysical compact objects such as neutron stars, whose spin frequency can be as high as $700 \mathrm{~Hz}$ [9].

We begin, in Sec. II, by discussing some aspects of the quantization of nonminimally coupled free scalar fields

\footnotetext{
*rfpm@ift.unesp.br

matsas@ift.unesp.br

*vanzella@ifsc.usp.br
}

containing unstable modes in a background which is flat in the asymptotic past and stationary and axially symmetric in the future. In Sec. III we present a simple general argument that shows that the parameter space which characterizes the instability is not modified at first order in the compact object's angular momentum. Then, we investigate secondorder deviations from staticity in a particular model, taking as the source of the gravitational field a class of slowly spinning shells. The general properties of the shell spacetime are presented in Sec. IV. Considering spinning thin shells allows us to push the analytical treatment further and arrive at clear conclusions about the role played by rotation on the instability. This is pursued in Sec. V. Section VI is devoted to a discussion of the results and to our final remarks. We assume metric signature $(-+++)$ and natural units in which $c=G=\hbar=1$ unless stated otherwise.

\section{AWAKING THE VACUUM OF NONMINIMALLY COUPLED SCALAR FIELDS IN THE SPACETIME OF ROTATING OBJECTS}

Let us consider a spacetime which is nearly flat in the asymptotic past and stationary and axially symmetric in the asymptotic future corresponding to the formation of a rotating compact object from originally low density matter. In particular, let us assume that in the future the spacetime is well described by the line element [10]

$$
\begin{aligned}
d s^{2}= & g_{00} d t^{2}+2 g_{03} d t d \varphi+g_{33} d \varphi^{2}+g_{11}\left(d x^{1}\right)^{2} \\
& +g_{22}\left(d x^{2}\right)^{2},
\end{aligned}
$$

where $g_{\mu \nu}=g_{\mu \nu}\left(x^{1}, x^{2}\right)$ is assumed to be smooth at the origin and continuous in the entire domain, while $\left(\partial_{t}\right)^{\mu}$ and $\left(\partial_{\varphi}\right)^{\mu}$ are (commuting) timelike and spacelike Killing fields, respectively. Moreover, the whole spacetime is 
assumed to be asymptotically flat and to bear no event or Cauchy horizons.

In this fixed background, let us consider the massless Klein-Gordon equation,

$$
\left(-\nabla^{\mu} \nabla_{\mu}+\xi R\right) \phi=0,
$$

describing the dynamics of a nonminimally coupled real scalar field $\phi$, where $\xi \in \mathbb{R}$ and $R$ is the scalar curvature.

Now, let us restrict attention to the spacetime portion described by the metric (1) and consider the following solution of Eq. (2) compatible with the spacetime symmetries and regular at the symmetry axis:

$$
\phi_{\omega m}\left(t, x^{1}, x^{2}, \varphi\right)=e^{-i \omega t+i m \varphi} F_{\omega m}\left(x^{1}, x^{2}\right),
$$

where $\omega \in \mathbb{C}, m \in \mathbb{Z}, F_{\omega m}=F_{\omega m}\left(x^{1}, x^{2}\right)$ satisfies the differential equation

$$
\begin{aligned}
& \frac{1}{\sqrt{-g}} \frac{\partial}{\partial x^{p}}\left(g^{p q} \sqrt{-g} \frac{\partial F_{\omega m}}{\partial x^{q}}\right) \\
& +\left(\frac{\omega^{2}+m^{2} g_{00} / g_{33}+2 \omega m g_{03} / g_{33}}{-g_{00}+g_{03}^{2} / g_{33}}-\xi R\right) F_{\omega m}=0
\end{aligned}
$$

with $p, q \in\{1,2\}$ and $g \equiv \operatorname{det}\left(g_{\mu \nu}\right)$. Since the metric (1) is assumed to be asymptotically flat, the behavior of $F_{\omega m}\left(x^{1}, x^{2}\right)$ at spatial infinity is given by

$$
F_{\omega m}(r, \theta) \stackrel{r \rightarrow \infty}{\longrightarrow} \sum_{\lambda= \pm} \sum_{l=|m|}^{\infty} N_{\omega l m \lambda} P_{l}^{m}(\cos \theta) \frac{e^{i \lambda \omega r}}{r},
$$

where $N_{\omega l m \lambda}=$ const, $P_{l}^{m}(y)$ are associate Legendre polynomials, and we have chosen coordinates $\left\{x^{1}, x^{2}\right\}$ to reduce asymptotically to the spherical ones $\{r, \theta\}$. The constants $N_{\omega l m \lambda}$ are determined by the regularity condition at the symmetry axis up to an overall factor.

For $\Im(\omega) \neq 0$, only solutions $F_{\omega m}$ with

$$
\lambda \Im(\omega)>0
$$

will be physically acceptable so that modes (3) are well behaved at spatial infinity. This constrains the acceptable values of $\omega$ to those (if any) for which either $N_{\omega l m+}=0$ (in the case $\Im(\omega)<0$ ) or $N_{\omega l m-}=0$ (in the case $\Im(\omega)>0$ ):

$$
F_{\omega m}(r, \theta) \stackrel{r \rightarrow \infty}{\longrightarrow} \sum_{l=|m|}^{\infty} N_{\omega l m \lambda} P_{l}^{m}(\cos \theta) \frac{e^{i \lambda \omega r}}{r}
$$

where $\lambda \Im(\omega)>0$. It should be noted that Eq. (4) allows us to write the equality (up to an arbitrary multiplicative constant)

$$
F_{\omega m}^{*}\left(x^{1}, x^{2}\right)=F_{\omega^{*} m}\left(x^{1}, x^{2}\right) .
$$

Here, we are interested in the case where unstable modes of the form (3) exist. Classically, the existence of such unstable modes implies that generic linear perturbation out of the $\phi=0$ equilibrium configuration grows unboundedly in time. This unbounded amplification of classical linear perturbations indicates the breakdown of the test-field approximation, in which the field evolves in a fixed background, and implies that the nonlinear interaction between the field and gravity must be taken into account.

In Refs. $[1,2]$ it was shown that even in the absence of classical perturbations quantum mechanics provides a natural mechanism through which the instability settles in by means of the amplification of quantum vacuum fluctuations and, consequently, of the field's vacuum energy density. Indeed, in Ref. [4] we argue that if the initial mean field amplitude is much larger than $\sqrt{\hbar}$ a classical description of the instability is suitable but if it is of the order of $\sqrt{\hbar}$ then a quantum treatment should be employed. In what remains of this section, we discuss some aspects of the field quantization in the presence of unstable modes. (See, e.g., Refs. [11-13] for the quantization procedure in some stationary spacetimes for which the field is stable and Ref. [14] for a rigorous discussion on the quantization of unstable fields in globally static spacetimes.)

In the canonical quantization procedure (see, e.g., Refs. [15,16]) the field and the associated momentum density are promoted to operators satisfying usual commutation relations. The field operator can be expanded in a set of mode functions,

$$
\hat{\phi}=\int d \mu(\sigma)\left[\hat{a}_{\sigma} u_{\sigma}^{(+)}+\hat{a}_{\sigma}^{\dagger} u_{\sigma}^{(-)}\right]
$$

where $d \mu(\sigma)$ is a measure on the set of quantum numbers $\sigma$. The modes $u_{\sigma}^{(+)}$and $u_{\sigma}^{(-)}=\left(u_{\sigma}^{(+)}\right)^{*}$ are positive- and negative-norm solutions of Eq. (2), respectively, satisfying

$$
\left(u_{\sigma}^{( \pm)}, u_{\sigma^{\prime}}^{( \pm)}\right)_{K G}= \pm \delta\left(\sigma, \sigma^{\prime}\right) \quad \text { and } \quad\left(u_{\sigma}^{( \pm)}, u_{\sigma^{\prime}}^{(\mp)}\right)_{K G}=0
$$

where the Klein-Gordon inner product $(,)_{K G}$ is defined by

$$
(u, v)_{K G} \equiv i \int_{\Sigma_{t}} d \Sigma n^{\mu}\left[u^{*} \nabla_{\mu} v-v \nabla_{\mu} u^{*}\right]
$$

with $\Sigma_{t}$ denoting a Cauchy surface with proper volume element $d \Sigma$ and future-pointing unit normal vector field $n^{\mu}$. The operator-valued coefficients in Eq. (9) satisfy $\left[\hat{a}_{\sigma}, \hat{a}_{\sigma^{\prime}}^{\dagger}\right]=\delta\left(\sigma, \sigma^{\prime}\right)$ and zero for the remaining commutators. The vacuum $|0\rangle$ associated with this representation is then defined by requiring $\hat{a}_{\sigma}|0\rangle=0$ for all $\sigma$. 
Let us assume the quantum state to be the vacuum $\left|0_{\text {in }}\right\rangle$ defined with respect to a basis $\left\{u_{\vec{k}}^{( \pm)}\right\}$of modes which behave as plane waves in the asymptotic past (where the spacetime is flat),

$$
u_{\vec{k}}^{( \pm)} \stackrel{\text { past }}{\sim}\left(16 \pi^{3}|\vec{k}|\right)^{-1 / 2} \exp [\mp i(|\vec{k}| t-\vec{k} \cdot \vec{x})], \quad \vec{k} \in \mathbb{R}^{3},
$$

with $(t, \vec{x})$ being usual Cartesian coordinates. Thus, $\left|0_{\text {in }}\right\rangle$ is the no-particle state according to static past observers.

Let us now construct another set of orthonormal modes defined by their behavior in the asymptotic future. We choose $\Sigma_{t}$ to be a $t=$ const hypersurface with normal vector field $n^{\mu}=(1 / N)(1,0,0, \Omega)$, where $N \equiv$ $\left(-g_{00}+g_{03}^{2} / g_{33}\right)^{1 / 2}$ and $\Omega \equiv-g_{03} / g_{33}$. For this purpose, we first point out a useful property for solutions of Eq. (4) with proper boundary conditions. From Eq. (4), we have

$$
\begin{aligned}
& \frac{\partial}{\partial x^{p}}\left[g^{p q} \sqrt{-g}\left(F_{\omega^{\prime} m} \frac{\partial F_{\omega m}^{*}}{\partial x^{q}}-F_{\omega m}^{*} \frac{\partial F_{\omega^{\prime} m}}{\partial x^{q}}\right)\right] \\
& =\frac{\sqrt{-g}}{N^{2}}\left(\omega^{\prime}-\omega^{*}\right)\left(\omega^{*}+\omega^{\prime}-2 m \Omega\right) F_{\omega m}^{*} F_{\omega^{\prime} m} .
\end{aligned}
$$

Integrating Eq. (13) by recalling Eqs. (5)-(8), a nontrivial weighted orthonormality relation for $F_{\omega m}$ can be obtained:

$$
\int d x^{1} d x^{2} \frac{\sqrt{-g}}{N^{2}}\left(\omega+\omega^{\prime}-2 m \Omega\right) F_{\omega m} F_{\omega^{\prime} m}=2 \omega \delta_{\omega \omega^{\prime}},
$$

for $\omega, \omega^{\prime} \in \mathbb{C}-\mathbb{R}$ and

$$
\int d x^{1} d x^{2} \frac{\sqrt{-g}}{N^{2}}\left(\omega+\omega^{\prime}-2 m \Omega\right) F_{\omega m}^{*} F_{\omega^{\prime} m}=2 \omega \delta\left(\omega-\omega^{\prime}\right)
$$

for $\omega, \omega^{\prime} \in \mathbb{R}$.

Now, we can construct a set of orthonormal solutions of Eq. (2) by determining their behavior at the asymptotic future. This set can in principle comprise both timeoscillatory (stationary) and tachyonic (nonstationary) modes. Positive-norm oscillatory modes read

$$
v_{\omega m}^{(+)} \stackrel{\text { future }}{\sim} \frac{e^{-i \omega t+i m \varphi}}{(4 \pi \omega)^{1 / 2}} F_{\omega m}\left(x^{1}, x^{2}\right)
$$

with $\omega>0$, while positive-norm tachyonic modes read

$$
\begin{aligned}
w_{\omega m}^{(+)} & \stackrel{\text { future }}{\sim} \sec (\alpha-\beta)^{1 / 2} \\
& \times\left[(8 \pi \omega)^{-1 / 2} e^{-i \omega t+i m \varphi} e^{i \alpha} F_{\omega m}\left(x^{1}, x^{2}\right)\right. \\
& \left.+\left(8 \pi \omega^{*}\right)^{-1 / 2} e^{-i \omega^{*} t+i m \varphi} e^{i \beta} F_{\omega m}^{*}\left(x^{1}, x^{2}\right)\right],
\end{aligned}
$$

with $\Im(\omega)>0$ (see Eq. (7); the principal square root is assumed). We note that by setting $\alpha=-\beta=\pi / 6$, Eq. (17) matches the form presented in Refs. [1,2] for the static case where $\omega$ is purely imaginary. It can be verified that the set $\left\{v_{\omega m}^{( \pm)}, w_{\omega m}^{( \pm)}\right\}$characterized by the asymptotic forms (16) and (17) is orthonormalized in agreement with Eq. (10).

The existence of tachyonic modes (17) implies that at least some of the in-modes (12) will go through a phase of exponential growth and, consequently, for a field in the in-vacuum state $\left|0_{\text {in }}\right\rangle$, the expectation value of $\hat{\phi}^{2}$ will be exponentially amplified in time,

$$
\left\langle 0_{\text {in }}\left|\hat{\phi}^{2}\right| 0_{\text {in }}\right\rangle \stackrel{\text { future }}{\sim} \frac{\hbar \kappa}{4 \pi|\bar{\omega}|} e^{2 \Im(\bar{\omega}) t}\left|F_{\bar{\omega} \bar{m}}\right|^{2}\left[1+O\left(e^{-\epsilon t}\right)\right],
$$

(although $\left\langle 0_{\text {in }}|\hat{\phi}| 0_{\text {in }}\right\rangle=0$ ). Here, $\kappa \sim 1$ encodes information about the transition to the unstable phase, $\epsilon$ is some positive constant, $\bar{\omega}$ is the $\omega$ with largest value of $\Im(\omega)$ (achieved for a certain value of $m=\bar{m}$ ), and we have restored the $\hbar$. This amplification of vacuum fluctuations leads to an exponential enhancement of the expectation value of the field's stress-energy-momentum tensor, as was discussed in Ref. [1]. The system then evolves according to Einstein's semiclassical equations, at least while fluctuations of the field's stress-energy-momentum tensor are relatively "small" [17].

In this paper, we will focus on searching for solutions in the form (3) with $\Im(\omega)>0$, which are regular at the origin and vanish at spatial infinity [see Eq. (7), where $\lambda=+$ ]. Normalized tachyonic modes can be constructed from these solutions by adjusting the normalization as in Eq. (14). Our main purpose will be to understand how the range of field couplings $\xi$ for which unstable modes appear changes due to rotation.

\section{FIRST-ORDER DEVIATIONS FROM STATICITY}

Here, we argue that in order to extract nontrivial results concerning the instability analysis, we must go beyond first-order deviations from staticity. First, let us assume that the metric components in Eq. (1) are analytic functions of $J / M^{2}$ so that a perturbative treatment for small $J / M^{2}$ is meaningful, where $M$ and $J$ are mass and angular momentum of the compact object (computed, e.g., by Komar formulas).

Physically, it is clear that the field instability cannot depend on the rotation direction. Mathematically, this can be seen as follows. First, we note that rotation reversal, $J \rightarrow-J$, is equivalent to time reversal, $t \rightarrow-t$. This implies that $g_{03}$ is an odd function of $J$, while the remaining metric components, as well as $R$, are even. Then, for every regular solution $F_{\omega m}^{(\xi, J, M, \ldots)}$ of Eq. (4) there will exist a corresponding one 


$$
F_{\omega-m}^{(\xi,-J, M, \ldots)} \propto F_{\omega m}^{(\xi, J, M, \ldots)},
$$

where $(\xi, J, M, \ldots)$ was added to explicitly label all field and spacetime parameters on which $F_{\omega m}$ depends. Since modes with all values of $m$ enter in the field expansion, we conclude that whenever we have instability for a configuration $(\xi, J, M, \ldots)$ the same will be true for $(\xi,-J, M, \ldots)$. In particular,

$$
\xi_{0}(J, M, \ldots)=\xi_{0}(-J, M, \ldots),
$$

where $\xi_{0}=\xi_{0}(J, M, \ldots)$ is the value of $\xi$ which marks the appearance of (any) tachyonic modes as a function of the spacetime parameters. As a result, in order to see effects due to rotation in $\xi_{0}$, we must carry out our expansion at least up to second order in $J / M^{2}$.

Much less intuitive is the fact that

$$
\xi_{0 ; m, \Im(\omega)}(J, M, \ldots)=\xi_{0 ; m, \Im(\omega)}(-J, M, \ldots),
$$

where $\xi_{0 ; m, \Im(\omega)}=\xi_{0 ; m, \Im(\omega)}(J, M, \ldots)$ is the value of $\xi$ which marks the appearance of a tachyonic mode with quantum numbers $m$ and $\mathfrak{I}(\omega)$ (we have omitted $\mathfrak{R}(\omega)$, since it is irrelevant for the instability). The fact that $\xi_{0 ; m, \Im(\omega)}$ is an even function of $J / M^{2}$ can be traced back to the fact that if $F_{\omega m}^{(\xi, J, M, \ldots)}$ is a regular solution of Eq. (4), the same is true for $F_{-\omega^{*} m}^{(\xi,-J, \ldots)}$, since

$$
F_{\omega m}^{(\xi, J, M, \ldots) *} \propto F_{-\omega^{*}-m}^{(\xi, J, M, \ldots)} \propto F_{-\omega^{*} m}^{(\xi,-J, M, \ldots)} .
$$

From the discussion above, we conclude that corrections due to rotation to $\xi_{0}$ and $\xi_{0 ; m, \Im(\omega)}$ are of even order on the parameter $J / M^{2}$ (which will be manifest in the results of Sec. V). Thus, in what follows, we will explore secondorder corrections in a particular spacetime, which we now describe.

\section{ROTATING THIN SHELLS}

The Kerr metric, given in Boyer-Lindquist coordinates by

$$
\begin{aligned}
d s^{2}= & -\left(1-\frac{2 M r}{r^{2}+a^{2} \cos ^{2} \theta}\right) d t^{2}-\frac{4 a M r \sin ^{2} \theta}{r^{2}+a^{2} \cos ^{2} \theta} d t d \varphi \\
& +\left(r^{2}+a^{2} \cos ^{2} \theta\right)\left(\frac{d r^{2}}{r^{2}-2 M r+a^{2}}+d \theta^{2}\right) \\
& +\left(r^{2}+a^{2}+\frac{2 M r a^{2} \sin ^{2} \theta}{r^{2}+a^{2} \cos ^{2} \theta}\right) \sin ^{2} \theta d \varphi^{2},
\end{aligned}
$$

besides being the only vacuum solution of Einstein's equations describing stationary black holes, can in principle also approximate the gravitational field outside an axially symmetric rotating source with mass $M$ and angular momentum $J=a M$. In particular, in Ref. [18] a spinning shell was considered as a source of the Kerr metric and the matching of internal and external solutions was worked out explicitly up to third order in the rotation parameter. In this section, we will describe in some detail the particular case of a flat interior matched with an external Kerr field up to second order in $a / M$, which will suffice as a prototype model of a rotating system.

Therefore, let us consider a stationary and axially symmetric thin shell of matter surrounded by vacuum. The spacetime region internal to the shell is taken to be flat, with line element

$$
d s_{-}^{2}=-d \tau^{2}+d \rho^{2}+\rho^{2}\left(d \Theta^{2}+\sin ^{2} \Theta d \Phi^{2}\right),
$$

while the external-to-the-shell portion of the spacetime will be described by the Kerr metric, Eq. (19), expanded up to second order in $a / M$ :

$$
\begin{aligned}
d s_{+}^{2}= & -\left[1-\frac{2 M}{r}\left(1-\frac{a^{2}}{r^{2}} \cos ^{2} \theta\right)\right] d t^{2}-\frac{4 a M}{r} \sin ^{2} \theta d t d \varphi \\
& +\left(r^{2}+a^{2} \cos ^{2} \theta-\frac{a^{2} r^{2}}{r^{2}-2 M r}\right) \frac{d r^{2}}{r^{2}-2 M r} \\
& +\left[r^{2}+a^{2}\left(1+\frac{2 M}{r} \sin ^{2} \theta\right)\right] \sin ^{2} \theta d \varphi^{2} \\
& +\left(r^{2}+a^{2} \cos ^{2} \theta\right) d \theta^{2}
\end{aligned}
$$

This approximation is valid as long as the corresponding error is small, i.e., $g_{\mu \nu}^{\mathrm{kerr}}-g_{\mu \nu}^{\mathrm{approx}} \ll g_{\mu \nu}^{\mathrm{kerr}}$, which is satisfied if

$$
a^{2} \ll M^{2} \quad \text { and } \quad r \gg 2 M .
$$

The label "-" ("+") is used above and in what follows to indicate the restriction of certain quantities to the inner (outer) spacetime region with respect to the shell's world tube, which we denote by $\mathcal{S}$.

Equations (20) and (21) will represent portions of a single spacetime (with a singular three-dimensional timelike boundary $\mathcal{S}$ between them) provided that the internal and external metrics induced on $\mathcal{S}$, denoted by $h_{a b}$, coincide. Indeed, in Ref. [18], this was shown to be possible if $\mathcal{S}$ is determined by

$$
\left.r\right|_{\mathcal{S}}=r_{\mathcal{S}}(\theta) \equiv \mathrm{R}\left[1-\frac{a^{2} \mathrm{~F}^{2}}{\mathrm{R}^{2}} \cos ^{2} \theta\right],
$$

where $\mathrm{R}=$ const $>0$ is the shell equatorial radial coordinate and

$$
\mathrm{F} \equiv \sqrt{1-2 M / \mathrm{R}} .
$$

Note that since Eq. (21) is only reliable in the regime given by conditions (22), it is necessary that at least $\mathrm{R}>2 M$. It is convenient to cover $\mathcal{S}$ with coordinates $\zeta^{a}=(t, \theta, \varphi)$, 
$a=0,2,3$, since the shell lies at $r=r_{\mathcal{S}}(\theta)$ [see Eq. (23)]. Then, the continuity condition above allows us to relate the internal coordinates on $\mathcal{S}$ with $\zeta^{a}$ as

$$
\begin{aligned}
\left.\tau\right|_{\mathcal{S}} & =A t,\left.\quad \rho\right|_{\mathcal{S}}=\rho_{\mathcal{S}}(\theta), \\
\left.\Theta\right|_{\mathcal{S}} & =\Theta_{\mathcal{S}}(\theta),\left.\quad \Phi\right|_{\mathcal{S}}=\varphi-\tilde{\Omega} t,
\end{aligned}
$$

where

$$
\begin{aligned}
A & \equiv \mathrm{F}\left(1+\frac{2 a^{2} M^{2}}{\mathrm{R}^{4} \mathrm{~F}^{2}}\right), \\
\rho_{\mathcal{S}}(\theta) & \equiv \mathrm{R}\left[1+\frac{a^{2}}{2 \mathrm{R}^{2}}\left(1+\frac{2 M}{\mathrm{R}}-3 \cos ^{2} \theta\right)\right], \\
\Theta_{\mathcal{S}}(\theta) & \equiv \theta+\frac{a^{2}}{2 \mathrm{R}^{2}}\left(1+\frac{2 M}{\mathrm{R}}\right) \sin \theta \cos \theta, \\
\tilde{\Omega} & \equiv \frac{2 a M}{\mathrm{R}^{3}} .
\end{aligned}
$$

The shell can be shown to be slightly oblate according to zero-angular-momentum observers, since on a $t=$ const section of $\mathcal{S}$,

$$
\frac{L_{\text {equatorial }}}{L_{\text {meridional }}}=1+\frac{3 a^{2}}{4 \mathrm{R}^{2}} \geq 1
$$

where $L_{\text {equatorial }}$ and $L_{\text {meridional }}$ are the equatorial $(\theta=\pi / 2)$ and meridional ( $\varphi=$ const) shell proper lengths, respectively. Note also that in this approximation the shell rotates rigidly with angular velocity [18]

$$
\Omega_{\text {shell }}=d \varphi / d t=\tilde{\Omega}(1+2 \mathrm{~F})(1-\mathrm{F})^{-1}(1+3 \mathrm{~F})^{-1}
$$

as measured by static observers at infinity, where $\tilde{\Omega}$ was defined in Eq. (29).

Once the spacetime is determined, the stress-energymomentum tensor of the corresponding matter layer is also fixed (see, e.g., Ref. [19]),

$$
T^{\mu \nu}=S^{a b} e_{a}^{\mu} e_{b}^{\nu} \delta(\ell)
$$

where $\ell$ is the proper distance along geodesics which intercept $\mathcal{S}$ orthogonally (such that $\ell<0, \ell=0$, and $\ell>0$ inside, on, and outside $\mathcal{S}$, respectively), $e_{a}^{\mu} \equiv$ $\partial x^{\mu} / \partial \zeta^{a}$ are the components of the coordinate vectors $\partial / \partial \zeta^{a}=\left(\partial_{t}, \partial_{\theta}, \partial_{\varphi}\right)$ defined on $\mathcal{S}$, and

$$
S^{a b}=-\frac{1}{8 \pi}\left(\Delta K^{a b}-h^{a b} \Delta K\right)
$$

is the surface stress-energy-momentum tensor of the shell. Here, $K_{a b}$ is the extrinsic curvature, $K \equiv K_{a b} h^{a b}$, and $\Delta A^{a b c_{m n o . . .}}$ denotes the discontinuity of some quantity
$A^{a b c \ldots i n o \ldots}$ across $\mathcal{S}$. A direct calculation, following Ref. [18], leads to

$$
\begin{aligned}
8 \pi S_{0}{ }^{0}= & \frac{2 \mathrm{~F}}{\mathrm{R}}-\frac{2}{\mathrm{R}}-\frac{a^{2}}{\mathrm{R}^{3}}\left(2-2 \mathrm{~F}+\frac{M}{\mathrm{FR}}-\frac{2 M}{\mathrm{R}}\right) \\
& -\frac{3 a^{2}}{\mathrm{R}^{3}}\left(2 \mathrm{~F}-2+\frac{3 M \mathrm{~F}}{2 \mathrm{R}}-\frac{M}{2 \mathrm{FR}}-\frac{M^{2}}{\mathrm{FR}^{2}}\right) \cos ^{2} \theta,
\end{aligned}
$$

$$
\begin{aligned}
8 \pi S_{2}^{2}= & \frac{\mathrm{F}}{\mathrm{R}}+\frac{M}{\mathrm{FR}^{2}}-\frac{1}{\mathrm{R}}+\frac{a^{2}}{2 \mathrm{R}^{3}}\left(1-\frac{1}{\mathrm{~F}}+\frac{2 M}{\mathrm{R}}-\frac{M}{\mathrm{RF}^{3}}\right) \\
& +\frac{3 a^{2}}{2 \mathrm{R}^{3}}\left(1-\mathrm{F}-\frac{M \mathrm{~F}}{\mathrm{R}}\right) \cos ^{2} \theta, \\
8 \pi S_{3}^{3}= & \frac{\mathrm{F}}{\mathrm{R}}+\frac{M}{\mathrm{FR}^{2}}-\frac{1}{\mathrm{R}}+\frac{a^{2}}{\mathrm{R}^{3}}\left(2 \mathrm{~F}+\frac{1}{2 \mathrm{~F}}-\frac{5}{2}+\frac{M}{\mathrm{R}}\right. \\
& \left.+\frac{M \mathrm{~F}}{\mathrm{R}}+\frac{2 M^{2}}{\mathrm{FR}^{2}}-\frac{M}{2 \mathrm{RF}^{3}}\right)+\frac{a^{2}}{\mathrm{R}^{3}}\left(\frac{9}{2}-\frac{9 \mathrm{~F}}{2}-\frac{2 M}{\mathrm{FR}}\right. \\
& \left.-\frac{5 M \mathrm{~F}}{2 \mathrm{R}}-\frac{2 M^{2}}{\mathrm{FR}^{2}}\right) \cos ^{2} \theta, \\
8 \pi S_{0}^{3}= & -\frac{a M}{\mathrm{R}^{4}}\left(2+\frac{1}{\mathrm{~F}}\right), \quad 8 \pi S_{0}^{3}=\frac{3 M a}{\mathrm{FR}^{2}} \sin ^{2} \theta .
\end{aligned}
$$

A result that will be particularly useful later is

$$
\begin{aligned}
\Delta K= & \frac{2 \mathrm{~F}}{\mathrm{R}}+\frac{M}{\mathrm{FR}^{2}}-\frac{2}{\mathrm{R}}+\frac{a^{2}}{\mathrm{R}^{3}}\left(2 \mathrm{~F}-2+\frac{2 M}{\mathrm{R}}-\frac{M}{2 \mathrm{RF}^{3}}\right) \\
& +\frac{3 a^{2}}{\mathrm{R}^{3}}\left(2-2 \mathrm{~F}-\frac{3 M \mathrm{~F}}{2 \mathrm{R}}\right) \cos ^{2} \theta .
\end{aligned}
$$

It can be verified from the stress-energy-momentum tensor written above that the shell gravitational mass and angular momentum are indeed equal to $M$ and $a M$, respectively. Other physical properties can be more readily investigated through the eigenvalues of $S_{a b}$. For this purpose, let us define vectors $\hat{e}_{\alpha}^{a}$ that form an orthonormal basis on $\mathcal{S}$, in the sense that

$$
h_{a b} \hat{e}_{\alpha}^{a} \hat{e}_{\beta}^{b}=\eta_{\alpha \beta},
$$

where $\eta_{\alpha \beta}=\operatorname{diag}(-1,1,1)$. The surface density and pressures are then eigenvalues with respect to these vectors and can be computed to be

$$
\begin{aligned}
& \sigma=S_{a b} \hat{e}_{0}^{a} \hat{e}_{0}^{b}=-S_{0}^{0}+\frac{S_{0}{ }^{3} S_{3}{ }^{0}}{\left(S_{3}{ }^{3}\right)^{(0)}-\left(S_{0}{ }^{0}\right)^{(0)}}, \\
& p_{\theta}=S_{a b} \hat{e}_{2}^{a} \hat{e}_{2}^{b}=S_{2}^{2}, \\
& p_{\varphi}=S_{a b} \hat{e}_{3}^{a} \hat{e}_{3}^{b}=S_{3}{ }^{3}+\frac{S_{0}{ }^{3} S_{3}{ }^{0}}{\left(S_{3}{ }^{3}\right)^{(0)}-\left(S_{0}{ }^{0}\right)^{(0)}},
\end{aligned}
$$


MENDES, MATSAS, AND VANZELLA

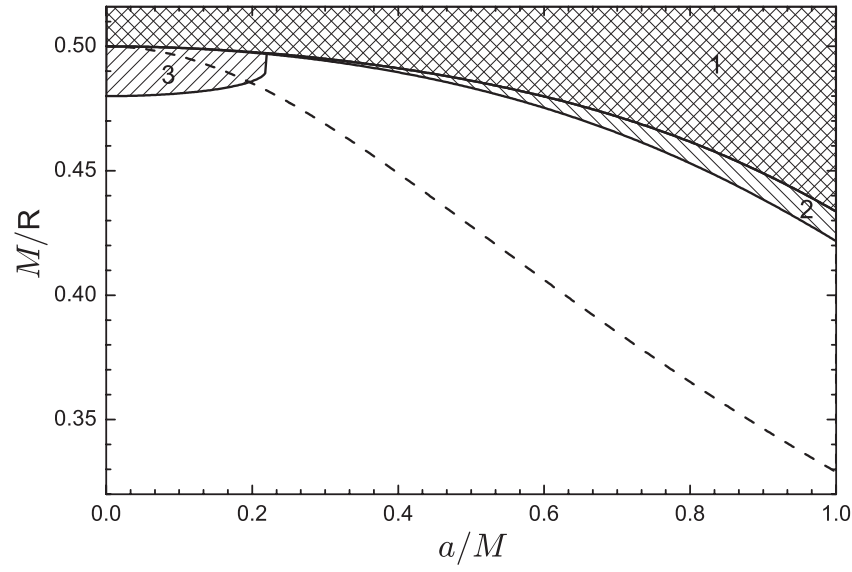

FIG. 1. The shaded region indicates the values of $M / \mathrm{R}$ which are excluded by the weak, strong or dominant energy conditions, in terms of the rotation parameter $a / M$. The weak, strong, and dominant energy conditions are not satisfied in region 1 , in the union of regions 1 and 2, and in the union of regions 1 and 3, respectively. When $a=0$, we get the static limit, in which case the dominant energy condition is violated for $M / \mathrm{R}>0.48$. The region below the dashed line corresponds to values of $M / \mathrm{R}$ where the error in approximating Eq. (19) by Eq. (21) is less than $10 \%$.

where we have introduced the superscript "(0)" in some terms to indicate that only the contributions of zeroth order in $a / M$ need to be considered to keep the approximation consistent up to second order.

The classical energy conditions can be explicitly stated in terms of these eigenvalues (see, e.g., Ref. [19]). The white region in Fig. 1 shows the values of $M / \mathrm{R}$ for which the weak, strong, and dominant energy conditions are satisfied as a function of $a / M$. The region below the dashed line indicates the values of $M / \mathrm{R}$ which comply with the condition

$$
\left(g_{\mu \nu}^{\mathrm{kerr}}-g_{\mu \nu}^{\mathrm{approx}}\right) / g_{\mu \nu}^{\mathrm{kerr}}<0.1 .
$$

In the subsequent analysis, we will only consider shells with $M / \mathrm{R}$ below this dashed line.

\section{SECOND-ORDER DEVIATIONS FROM STATICITY IN A SHELL MODEL}

In this section, we aim at investigating the influence of rotation on the parameter space that characterizes unstable configurations. We take as a model of a rotating system the slowly spinning shells described in Sec. IV and look for unstable solutions of Eq. (2) in this spacetime.

Let $\phi^{-}$and $\phi^{+}$denote solutions of $\nabla_{\mu} \nabla^{\mu} \phi^{ \pm}=0$ in the inner and outer spacetime portions with respect to the shell's world tube $\mathcal{S}$, respectively. They combine to form a solution of Eq. (2) in the entire spacetime provided that they are continuous at $\mathcal{S}$,
PHYSICAL REVIEW D 90, 044053 (2014)

$$
\left.\phi^{-}(\tau, \rho, \Theta, \Phi)\right|_{\mathcal{S}}=\left.\phi^{+}(t, r, \theta, \varphi)\right|_{\mathcal{S}}
$$

and their derivative along the direction orthogonal to the shell is discontinuous by a definite amount:

$$
\left.\Delta(d \phi / d \ell)\right|_{\mathcal{S}}=-\left.2 \xi \Delta K \phi\right|_{\mathcal{S}},
$$

which follows from Eq. (2) if we notice from Eq. (31) that

$$
R=-8 \pi T=-2 \Delta K \delta(\ell),
$$

where $\Delta K$ is given in Eq. (37). Equations (42) and (43), together with appropriate boundary conditions on $\phi^{ \pm}$, determine uniquely the joined solution.

In the interior of $\mathcal{S}$, solutions of the form (3) can be written as

$$
\phi_{\omega^{\prime} m^{\prime}}^{-}(\tau, \rho, \Theta, \Phi)=\sum_{l=\left|m^{\prime}\right|}^{\infty} N_{\omega^{\prime} l m^{\prime}}^{-} e^{-i \omega^{\prime} \tau} \chi_{\omega^{\prime} l}(\rho) Y_{l m^{\prime}}(\Theta, \Phi),
$$

where $\omega^{\prime} \in \mathbb{C}, m^{\prime} \in \mathbb{Z}, Y_{l m^{\prime}}(\Theta, \Phi)$ are spherical harmonics, $N_{\omega^{\prime} l m^{\prime}}^{-}$are arbitrary (complex) constants and $\chi_{\omega^{\prime} l}(\rho)$ satisfy the spherical Bessel equation,

$$
\rho^{2} \frac{d^{2} \chi_{\omega^{\prime} l}}{d \rho^{2}}+2 \rho \frac{d \chi_{\omega^{\prime} l}}{d \rho}+\left[\omega^{\prime 2} \rho^{2}-l(l+1)\right] \chi_{\omega^{\prime} l}=0,
$$

with the additional condition of regularity at the origin, so that, for $\omega^{\prime} \neq 0, \chi_{\omega^{\prime} l}(\rho) \propto j_{l}\left(\omega^{\prime} \rho\right)$. A summation is included in Eq. (44), since the spacetime is not spherically symmetric and, as we will show below, the matching in Eq. (42) will mix different values of $l$.

In the region external to $\mathcal{S}$, we analogously write

$$
\phi_{\omega m}^{+}(t, r, \theta, \varphi)=\sum_{l=|m|}^{\infty} N_{\omega l m}^{+} e^{-i \omega t+i m \varphi} \psi_{\omega l m}(r) S_{\omega l m}(\cos \theta),
$$

with $\omega \in \mathbb{C}$ and $m \in \mathbb{Z}$. Here, $S_{\omega l m}(\cos \theta)$ are spheroidal harmonics [20] satisfying

$$
\begin{aligned}
& \frac{1}{\sin \theta} \frac{d}{d \theta}\left(\sin \theta \frac{d S_{\omega l m}}{d \theta}\right) \\
& \quad+\left(\Lambda_{l m}+a^{2} \omega^{2} \cos ^{2} \theta-\frac{m^{2}}{\sin ^{2} \theta}\right) S_{\omega l m}=0,
\end{aligned}
$$

where

$$
\Lambda_{l m}=l(l+1)-\frac{\left(2 l^{2}+2 l-2 m^{2}-1\right) a^{2} \omega^{2}}{(2 l-1)(2 l+3)}+O\left(a^{4} \omega^{4}\right) .
$$


The expansion of $S_{\omega l m}(y)$ in powers of the dimensionless parameter $a \omega$ has the following leading terms:

$$
\begin{aligned}
S_{\omega l m}(y)= & P_{l}^{m}(y)+a^{2} \omega^{2}\left[-\frac{(l+m-1)(l+m)}{2(2 l+1)(2 l-1)^{2}} P_{l-2}^{m}(y)\right. \\
& \left.+\frac{(l-m+1)(l-m+2)}{2(2 l+1)(2 l+3)^{2}} P_{l+2}^{m}(y)\right]+O\left(a^{4} \omega^{4}\right) .
\end{aligned}
$$

The radial functions $\psi_{\omega l m}(r)$ obey the differential equation (up to second order)

$$
\begin{aligned}
& f\left(f+\frac{2 a^{2}}{r^{2}}\right) \frac{d^{2} \psi_{\omega l m}}{d r^{2}}+\frac{2}{r}\left(f+\frac{a^{2}}{r^{2}}\right)\left(1-\frac{M}{r}\right) \frac{d \psi_{\omega l m}}{d r} \\
& +\left[\omega^{2}\left(1+\frac{a^{2}}{r^{2}}+\frac{2 M a^{2}}{r^{3}}\right)-\frac{4 a M \omega m}{r^{3}}+\frac{m^{2} a^{2}}{r^{4}}\right. \\
& \left.\quad-\left(\frac{\Lambda_{l m} f}{r^{2}}+\frac{l(l+1) a^{2}}{r^{4}}\right)\right] \psi_{\omega l m}=0,
\end{aligned}
$$

where $f=f(r) \equiv 1-2 M / r$. Since we are looking for normalizable solutions with $\Im(\omega)>0, \psi_{\omega l m}(r)$ must go asymptotically as [see Eq. (7)]

$$
\psi_{\omega l m}(r) \stackrel{r \rightarrow+\infty}{\propto} \frac{e^{i \omega r}}{r}
$$

The continuity condition, Eq. (42), implies

$$
\begin{aligned}
& m^{\prime}=m, \\
& \omega^{\prime}=\frac{\omega-m \tilde{\Omega}}{A}=\frac{1}{\mathrm{~F}}\left(\omega-\frac{2 M m a}{\mathrm{R}^{3}}-\frac{2 \omega a^{2} M^{2}}{\mathrm{R}^{4} \mathrm{~F}^{2}}\right),
\end{aligned}
$$

and

$$
\begin{aligned}
& \sum_{l \geq|m|} N_{\omega l m}^{+} S_{\omega l m}(\cos \theta) \psi_{\omega l m}\left[r_{\mathcal{S}}(\theta)\right] \\
& =\sum_{l \geq|m|} N_{\omega^{\prime} l m}^{-} P_{l}^{m}\left[\cos \Theta_{\mathcal{S}}(\theta)\right] \chi_{\omega^{\prime} l}\left[\rho_{\mathcal{S}}(\theta)\right],
\end{aligned}
$$

where we recall that $\mathrm{F}=\sqrt{f(\mathrm{R})}$ [see Eq. (24)] and that $r_{\mathcal{S}}(\theta), \rho_{\mathcal{S}}(\theta)$, and $\Theta_{\mathcal{S}}(\theta)$ are defined in Eqs. (23), (27), and (28), respectively. Equation (50) comes from the spacetime axial symmetry, while Eq. (51) relates the energies ascribed to a certain mode by an inertial observer inside the shell with proper time $\tau$ and by a static observer at spatial infinity: $\omega$ and $\omega^{\prime}$ differ not only due to redshift but also due to the coupling between rotation and the mode's angular momentum.

Equation (52) can be used to express the coefficients $N_{\omega l m}^{+}$in terms of $N_{\omega^{\prime} l m}^{-}$. For this purpose, it will be useful to Taylor expand $\chi_{\omega^{\prime} l}\left[\rho_{\mathcal{S}}(\theta)\right]$ and $\psi_{\omega l m}\left[r_{\mathcal{S}}(\theta)\right]$ around $\rho=\mathrm{R}$ and $r=\mathrm{R}$, respectively [see Eqs. (27) and (23)], $\chi_{\omega^{\prime} l}\left[\rho_{\mathcal{S}}(\theta)\right]=\chi_{\omega^{\prime} l}(\mathrm{R})+\left.\frac{a^{2}}{2 \mathrm{R}}\left(1+\frac{2 M}{\mathrm{R}}-3 \cos ^{2} \theta\right) \frac{d \chi_{\omega^{\prime} l}^{(0)}(\rho)}{d \rho}\right|_{\mathrm{R}}$,

$$
\psi_{\omega l m}\left[r_{\mathcal{S}}(\theta)\right]=\psi_{\omega l m}(\mathrm{R})-\left.\frac{a^{2}}{\mathrm{R}} \mathrm{F}^{2} \cos ^{2} \theta \frac{d \psi_{\omega l m}^{(0)}(r)}{d r}\right|_{\mathrm{R}},
$$

and to fix $\chi_{\omega^{\prime} l}(\mathrm{R})=1$ and $\psi_{\omega l m}(\mathrm{R})=1$, which can be done with no loss of generality. The arbitrariness in the normalization of $\phi_{\omega^{\prime} m}^{-}$and $\phi_{\omega m}^{+}$will be completely encoded in $N_{\omega^{\prime} l m}^{-}$and $N_{\omega l m}^{+}$, which can be adjusted in order to comply with Eq. (14). Then, by writing

$$
N_{\omega^{\prime} l m}^{-}=N_{\omega^{\prime} l m}^{-(0)}+\frac{a^{2}}{M^{2}} N_{\omega^{\prime} l m}^{-(2)}
$$

and similarly for $N_{\omega l m}^{+}$, we note that for $a=0$, Eq. (52) implies $N_{\omega l m}^{+(0)}=N_{\omega^{\prime} l m}^{-(0)}$. Therefore, up to $O\left(a^{2} / M^{2}\right)$, we can write

$$
N_{\omega l m}^{+}=N_{\omega^{\prime} l m}^{-}+\frac{a^{2}}{M^{2}} g_{\omega^{\prime} l m}, \quad l \geq|m| .
$$

Inserting Eq. (56) and Eqs. (53)-(54) in Eq. (52), multiplying the latter by $P_{l^{\prime}}^{m}(\cos \theta)$ and integrating over $\theta$, we obtain, after some algebra,

$$
\begin{aligned}
g_{\omega^{\prime} l m}= & N_{\omega^{\prime} l m}^{-(0)} \alpha_{1}+N_{\omega^{\prime}(l+2) m}^{-(0)} \alpha_{2} \\
& +N_{\omega^{\prime}(l-2) m}^{-(0)} \alpha_{3} H(l-|m|-2),
\end{aligned}
$$

where $H(x)$ is the Heaviside step function,

$$
\begin{aligned}
\alpha_{1}= & \left.\frac{M^{2}}{\mathrm{R}}\left(\frac{M}{\mathrm{R}}-\frac{l^{2}+l-3 m^{2}}{(2 l+3)(2 l-1)}\right) \frac{d \chi_{\omega^{\prime} l}^{(0)}(\rho)}{d \rho}\right|_{\mathrm{R}} \\
& +\left.\frac{M^{2} \mathrm{~F}^{2}}{\mathrm{R}}\left(\frac{2 l^{2}+2 l-1-2 m^{2}}{(2 l+3)(2 l-1)}\right) \frac{d \psi_{\omega l m}^{(0)}(r)}{d r}\right|_{\mathrm{R}} \\
& -\frac{M^{2}}{2 \mathrm{R}^{2}}\left(\frac{l^{2}+l-3 m^{2}}{(2 l+3)(2 l-1)}\right)\left(1+\frac{2 M}{\mathrm{R}}\right), \\
\alpha_{2}= & \frac{M^{2}}{\mathrm{R}}\left(\frac{(l+m+1)(l+m+2)}{(2 l+3)(2 l+5)}\right) \\
& \times\left[-\frac{l+3}{2 \mathrm{R}}\left(1+\frac{2 M}{\mathrm{R}}\right)+\frac{\omega^{2} \mathrm{R}}{4 l+6}+\left.\mathrm{F}^{2} \frac{d \psi_{\omega(l+2) m}^{(0)}(r)}{d r}\right|_{\mathrm{R}}\right. \\
& \left.-\left.\frac{3}{2} \frac{d \chi_{\omega^{\prime}(l+2)}^{(0)}(\rho)}{d \rho}\right|_{\mathrm{R}}\right],
\end{aligned}
$$

and 


$$
\begin{aligned}
\alpha_{3}= & \frac{M^{2}}{\mathrm{R}}\left(\frac{(l-m-1)(l-m)}{(2 l-1)(2 l-3)}\right)\left[\frac{l-2}{2 \mathrm{R}}\left(1+\frac{2 M}{\mathrm{R}}\right)\right. \\
& \left.-\frac{\omega^{2} \mathrm{R}}{4 l-2}+\left.\mathrm{F}^{2} \frac{d \varphi_{\omega(l-2) m}^{(0)}(r)}{d r}\right|_{\mathrm{R}}-\left.\frac{3}{2} \frac{d \chi_{\omega^{\prime}(l-2)}^{(0)}(\rho)}{d \rho}\right|_{\mathrm{R}}\right] .
\end{aligned}
$$

It is worthwhile to note in Eq. (57) the coupling between multipolar indices $l$ and $l \pm 2$ that appears in $O\left(a^{2} / M^{2}\right)$ due to the absence of spherical symmetry [see Eq. (30)]. More generic deviations from spherical symmetry, such as those considered in Ref. [8], can give rise to a more involved mixing.

The discontinuity condition on the derivatives, Eq. (43), can be more explicitly written as

$$
\begin{aligned}
& \sum_{l \geq|m|} N_{\omega l m}^{+}\left[\left.\left.\frac{d r}{d \ell}\right|_{\mathcal{S}} \frac{d \psi_{\omega l m}(r)}{d r}\right|_{r_{\mathcal{S}}} S_{\omega l m}(\cos \theta)+\left.\frac{d \theta}{d \ell}\right|_{\mathcal{S}} \frac{d S_{\omega l m}(\cos \theta)}{d \theta} \psi_{\omega l m}\left(r_{\mathcal{S}}\right)\right] \\
& \quad-\sum_{l \geq|m|} N_{\omega^{\prime} l m}^{-}\left[\frac{d \rho}{d \ell}\left|\frac{d \chi_{\omega^{\prime} l}(\rho)}{d \rho}\right|_{\rho_{\mathcal{S}}} P_{l}^{m}\left(\cos \Theta_{\mathcal{S}}\right)+\left.\left.\frac{d \Theta}{d \ell}\right|_{\mathcal{S}} \frac{d P_{l}^{m}(\cos \Theta)}{d \Theta}\right|_{\Theta_{\mathcal{S}}} \chi_{\omega^{\prime} l}\left(\rho_{\mathcal{S}}\right)\right] \\
& =-2 \xi \Delta K \sum_{l \geq|m|} N_{\omega^{\prime} l m}^{-} \chi_{\omega^{\prime} l}\left(\rho_{\mathcal{S}}\right) P_{l}^{m}\left(\cos \Theta_{\mathcal{S}}\right),
\end{aligned}
$$

where the $\theta$ dependence has been omitted in several terms and

$\left.\frac{d r}{d \ell}\right|_{\mathcal{S}}=\mathrm{F}-\frac{a^{2} \mathrm{~F}}{2 \mathrm{R}^{2}}\left(1+\frac{2 M}{\mathrm{R}}\right) \cos ^{2} \theta+\frac{a^{2}}{2 \mathrm{R}^{2} \mathrm{~F}},\left.\quad \frac{d \rho}{d \ell}\right|_{\mathcal{S}}=1$, $\left.\frac{d \theta}{d \ell}\right|_{\mathcal{S}}=-\frac{2 a^{2} \mathrm{~F}}{\mathrm{R}^{3}} \sin \theta \cos \theta,\left.\quad \frac{d \Theta}{d \ell}\right|_{\mathcal{S}}=-\frac{3 a^{2}}{\mathrm{R}^{3}} \sin \theta \cos \theta$

are components of the unit vector field normal to $\mathcal{S}$. We can manipulate Eq. (58) in order to obtain a more enlightening expression. For this purpose, we make use of Eqs. (56)-(57), as well as Taylor expansions of $d \chi_{\omega^{\prime} l}(\rho) /\left.d \rho\right|_{\rho=\rho_{\mathcal{S}}}$ and $d \psi_{\omega l m}(r) /\left.d r\right|_{r=r_{S}}$ around $\rho=\mathrm{R}$ and $r=\mathrm{R}$ [analogous to Eqs. (53) and (54)]. Then, by multiplying Eq. (58) by $P_{l^{\prime}}^{m}(\cos \theta)$ and integrating over $\theta$, we can cast the resulting equation in the following form:

$$
\begin{aligned}
& \beta_{0}^{l} N_{\omega^{\prime} l m}^{-}+\frac{a^{2}}{M^{2}}\left[\beta_{1}^{l} N_{\omega^{\prime} l m}^{-(0)}+\beta_{2}^{l} N_{\omega^{\prime}(l+2) m}^{-(0)}\right. \\
& \left.+\beta_{3}^{l} N_{\omega^{\prime}(l-2) m}^{-(0)} H(l-|m|-2)\right]=0, \quad l \geq|m|,
\end{aligned}
$$

where $\beta_{j}^{l}, j \in\{0,1,2,3\}$, are coefficients which depend in principle on all mode and spacetime parameters except on $a$ (and which we avoid writing explicitly because of space restrictions). Then, in zeroth order in the rotation parameter, Eq. (59) reduces to

$$
\beta_{0}^{l} N_{\omega^{\prime} l m}^{-(0)}=0,
$$

which gives rise to a nontrivial solution for $\phi_{\omega^{\prime} m}^{-}$[see Eq. (44)] if

$$
\beta_{0}^{l_{0}}=0
$$

for some $l=l_{0} \geq|m|$. In this case, $N_{\omega^{\prime} l_{0} m}^{-(0)} \neq 0$ is fixed by the Klein-Gordon normalization while $N_{\omega^{\prime} l m}^{-(0)}=0$ for $l \neq l_{0}$ :

$$
N_{\omega^{\prime} l m}^{-(0)}=N_{\omega^{\prime} l_{0} m}^{-(0)} \delta_{l l_{0}}
$$

Condition (61) can be written as

$$
\xi^{(0)}=\left(\frac{4 \mathrm{~F}}{\mathrm{R}}+\frac{2 M}{\mathrm{FR}^{2}}-\frac{4}{\mathrm{R}}\right)^{-1}\left(\left.\frac{d \chi_{\omega^{\prime} l_{0}}^{(0)}}{d \rho}\right|_{\mathrm{R}}-\left.\mathrm{F} \frac{d \psi_{\omega l_{0} m}^{(0)}}{d r}\right|_{\mathrm{R}}\right),
$$

which expresses the value of $\xi$ that the field must have in order that unstable modes with quantum numbers $\omega^{\prime}$ $(=\omega / F), l_{0}$ and $m$ do exist in the spacetime of a static spherical shell with mass-to-radius ratio $M / \mathrm{R}$. In second order in $a / M$, Eq. (59) yields

$$
\begin{aligned}
& \beta_{0}^{l} N_{\omega^{\prime} l m}^{-(2)}+\beta_{1}^{l} N_{\omega^{\prime} l m}^{-(0)}+\beta_{2}^{l} N_{\omega^{\prime}(l+2) m}^{-(0)} \\
& \quad+\beta_{3}^{l} N_{\omega^{\prime}(l-2) m}^{-(0)} H(l-|m|-2)=0, \quad l \geq|m| .
\end{aligned}
$$

For $|m| \leq l \neq l_{0}$, Eq. (64) can be solved for $N_{\omega^{\prime} l m}^{-(2)}$,

$$
N_{\omega^{\prime} l m}^{-(2)}=-N_{\omega^{\prime} l_{0} m}^{-(0)}\left(\frac{\beta_{2}^{l_{0}-2}}{\beta_{0}^{l_{0}-2}} \delta_{l l_{0}-2}+\frac{\beta_{3}^{l_{0}+2}}{\beta_{0}^{l_{0}+2}} \delta_{l l_{0}+2}\right),
$$

which together with Eq. (62) determine $N_{\omega^{\prime} l m}^{-}$in Eq. (55) (with $N_{\omega^{\prime} l_{0} m}^{-(0)}$ fixed by normalization). Now, by using Eqs. (62) and (65) in Eq. (59), we obtain

$$
\beta_{0}^{l_{0}}+\frac{a^{2}}{M^{2}} \beta_{1}^{l_{0}}=0,
$$


which can be explicitly written as

$$
\begin{aligned}
\xi\left(\frac{4 \mathrm{~F}}{\mathrm{R}}+\frac{2 M}{\mathrm{FR}^{2}}-\frac{4}{\mathrm{R}}\right)\left[1+\frac{a^{2}}{2 \mathrm{R}^{2}} \frac{4 \mathrm{RF}^{4}-4 \mathrm{RF}^{3}+4 M \mathrm{~F}^{3}-M}{2 \mathrm{RF}^{4}+M \mathrm{~F}^{2}-2 \mathrm{RF}^{3}}+\frac{a^{2}}{2 \mathrm{R}^{2}} \frac{2 l_{0}^{2}+2 l_{0}-1-2 m^{2}}{\left(2 l_{0}+3\right)\left(2 l_{0}-1\right)} \frac{12 \mathrm{RF}-12 \mathrm{RF}^{2}-9 M \mathrm{~F}^{2}}{2 \mathrm{RF}^{2}+M-2 \mathrm{RF}}\right. \\
\left.+\left.\frac{a^{2}}{\mathrm{R}}\left(\frac{M}{\mathrm{R}}-\frac{l_{0}^{2}+l_{0}-3 m^{2}}{\left(2 l_{0}+3\right)\left(2 l_{0}-1\right)}\right) \frac{d \chi_{\omega^{\prime} l_{0}}^{(0)}}{d \rho}\right|_{\mathrm{R}}-\frac{a^{2}}{2 \mathrm{R}^{2}}\left(1+\frac{2 M}{\mathrm{R}}\right) \frac{l_{0}^{2}+l_{0}-3 m^{2}}{\left(2 l_{0}+3\right)\left(2 l_{0}-1\right)}\right]=-\left.\mathrm{F} \frac{d \psi \omega l_{0} m}{d r}\right|_{\mathrm{R}}+\left.\frac{d \chi_{\omega^{\prime} l_{0}}}{d \rho}\right|_{\mathrm{R}} \\
-\left.\frac{a^{2} \mathrm{~F}}{\mathrm{R}^{2}}\left[\alpha_{1} \mathrm{R}^{2}-\frac{2 l_{0}^{2}+2 l_{0}-1-2 m^{2}}{2\left(2 l_{0}+3\right)\left(2 l_{0}-1\right)}\left(1+\frac{2 M}{\mathrm{R}}\right)+\frac{1}{2 \mathrm{~F}^{2}}\right] \frac{d \psi_{\omega l_{0} m}^{(0)}}{d r}\right|_{\mathrm{R}}+\frac{a^{2} \mathrm{~F}^{3}}{\mathrm{R}} \frac{2 l_{0}^{2}+2 l_{0}-1-2 m^{2}}{\left(2 l_{0}+3\right)\left(2 l_{0}-1\right)} \frac{\left.d^{2} \psi_{\omega l_{0} m}^{(0)}\right|_{\mathrm{R}}}{d r^{2}} \\
+\left.\frac{a^{2}}{\mathrm{R}}\left(\frac{M}{\mathrm{R}}-\frac{l_{0}^{2}+l_{0}-3 m^{2}}{\left(2 l_{0}+3\right)\left(2 l_{0}-1\right)}\right) \frac{d^{2} \chi_{\omega^{\prime} l_{0}}^{(0)}}{d \rho^{2}}\right|_{\mathrm{R}}-\frac{a^{2}}{2 \mathrm{R}^{2}} \frac{l_{0}^{2}+l_{0}-3 m^{2}}{\left(2 l_{0}-1\right)\left(2 l_{0}+3\right)}\left[\frac{4 \mathrm{~F}}{\mathrm{R}}-\frac{6}{\mathrm{R}}+\left.\left(1+\frac{2 M}{\mathrm{R}}\right) \frac{d \chi_{\omega^{\prime} l_{0}}^{(0)}}{d \rho}\right|_{\mathrm{R}}\right] .
\end{aligned}
$$

If we write $\omega=\omega_{R}+i \omega_{I}$, then, for each set $\left\{\omega_{I}, l_{0}, m, M / \mathrm{R}, a / M\right\}$ of parameters, Eq. (67) is a complex equation, the imaginary part of which can be solved for $\omega_{R}$, and the real part of which then returns a value for $\xi$. Then, for each fixed $l_{0} \geq|m|, \xi$ is the field coupling that marks the appearance of unstable terms ("partial modes") in the sum in Eq. (44) with quantum numbers $\omega^{\prime}$ and $m$, and with $N_{\omega^{\prime} l m}^{-}$given by Eq. (55) with Eqs. (62) and (65) [the corresponding term in the exterior of $\mathcal{S}$ is determined from Eq. (46) together with Eqs. (56) and (57)]. The above procedure to calculate $\xi$ relies on the knowledge of $\psi_{\omega l m}(r)$, which we compute numerically by integrating Eq. (48) subject to the boundary condition (49) and normalization condition $\psi_{\omega l m}(\mathrm{R})=1$ [see discussion below Eq. (54)]. In Fig. 2 this method is employed to obtain the values of $\xi$ and $M / \mathrm{R}$ which trigger the instability for $a / M=0.2, l_{0}=m=0$ and $0<\omega_{I} \leq 0.4$. We note from Fig. 2 that the external boundaries of the unstable regions (black curves) are numerically consistent with $\omega_{I}=\omega_{R}=0$, which is compatible with the general result [21] that instability sets in through zero-frequency modes [22]. Therefore, let us now specialize to $\omega=0$, in which case we can obtain analytically the second-order approximation for the implicit functions in Eq. (67). Thus, we write up to second order

$$
\chi_{\omega_{0}^{\prime} l}(\rho)=\chi_{\omega_{0}^{\prime} l}^{(0)}(\rho)+\frac{a^{2}}{M^{2}} \chi_{\omega_{0}^{\prime l} l}^{(2)}(\rho),
$$

where $\omega_{0}^{\prime} \equiv-m \tilde{\Omega} / \mathrm{F}$ is the $\omega^{\prime}$ frequency when $\omega=0$ and

$$
\psi_{0 l m}(r)=\psi_{0 l m}^{(0)}(r)+\frac{a^{2}}{M^{2}} \psi_{0 l m}^{(2)}(r)
$$

The static limit is straightforward:

$$
\chi_{\omega_{0}^{\prime} l}^{(0)}(\rho)=\frac{\rho^{l}}{\mathrm{R}^{l}}, \quad \psi_{0 l m}^{(0)}(r)=\frac{Q_{l}(r / M-1)}{Q_{l}(\mathrm{R} / M-1)},
$$

where $Q_{l}(x)$ is the Legendre function of the second kind. The functions $\chi_{\omega_{0}^{\prime} l}^{(2)}(\rho)$ and $\psi_{0 l m}^{(2)}(r)$ satisfy inhomogeneous differential equations, for which the homogeneous part may be solved in terms of simple special functions. Therefore, standard methods (see, e.g., Ref. [23]) can be used in order to derive the full expressions (68) and (69). In particular, we obtain

$$
\left.\frac{d \chi_{\omega_{0}^{\prime} l}}{d \rho}\right|_{\mathrm{R}}=\left.\frac{d \chi_{\omega_{0}^{\prime} l}^{(0)}}{d \rho}\right|_{\mathrm{R}}-\frac{4 a^{2} M^{2} m^{2}}{\mathrm{R}^{5} \mathrm{~F}^{2}(3+2 l)}
$$

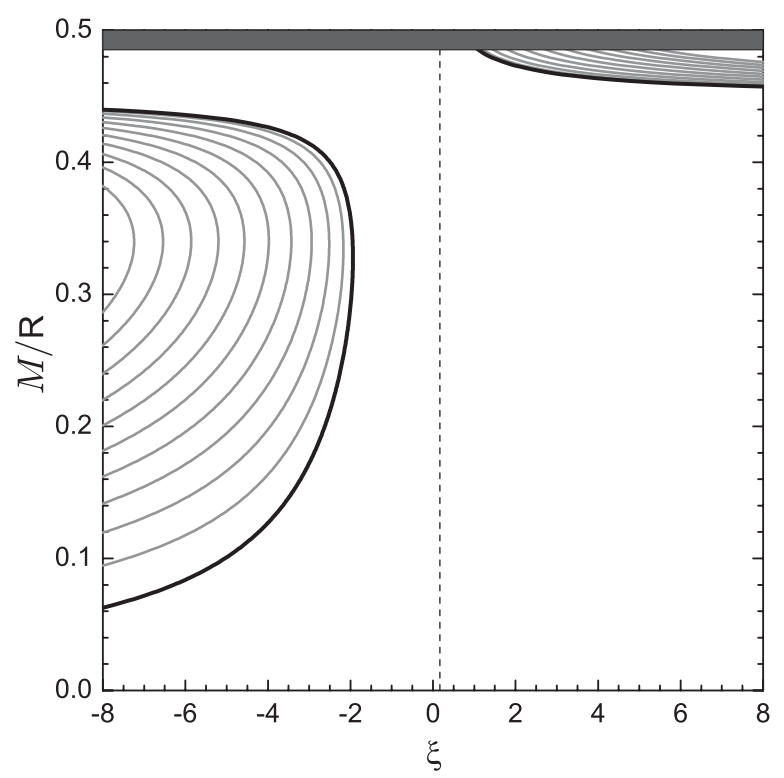

FIG. 2. Diagram showing the regions in the parameter space $(\xi, M / \mathrm{R})$ for which the instability is triggered when $a / M=0.2$. The black curves are characterized by $\omega_{I} \approx 0$ and provide the boundary of the unstable regions. Internal gray curves are characterized by values of $\omega_{I}$ which increase in steps of 0.04 up to $\omega_{I}=0.4$. Here, we have set $l_{0}=m=0$. The black strip excludes values of $M / \mathrm{R}$ for which Eq. (41) does not hold and the vertical dashed line indicates the conformal-coupling value $\xi=1 / 6$. 
and

$$
\begin{aligned}
\left.\frac{d \psi_{0 l m}}{d r}\right|_{\mathrm{R}}= & \left.\frac{d \psi_{0 l m}^{(0)}}{d r}\right|_{\mathrm{R}}+\frac{a^{2} C_{l}}{M^{2} Q_{l}(\mathrm{R} / M-1)}\left[\left.\frac{d P_{l}(r / M-1)}{d r}\right|_{\mathrm{R}}\right. \\
& \left.-\left.\frac{P_{l}(\mathrm{R} / M-1)}{Q_{l}(\mathrm{R} / M-1)} \frac{d Q_{l}(r / M-1)}{d r}\right|_{\mathrm{R}}\right],
\end{aligned}
$$

where

$$
\begin{aligned}
C_{l} \equiv & \int_{1}^{\infty} d x Q_{l}(x \mathrm{R} / M-1)\left[\left.\frac{M}{\mathrm{R}} \frac{d^{2} Q_{l}(t \mathrm{R} / M-1)}{d t^{2}}\right|_{t=x}\right. \\
& \left.+\frac{m^{2}}{x^{2} \mathrm{R} / M-2 x} Q_{l}(x \mathrm{R} / M-1)\right] .
\end{aligned}
$$

Therefore, by plugging Eqs. (70), (71), and (72) into Eq. (67), we obtain an analytical expression [except for the simple integral of Eq. (73)] which can be directly solved for $\xi$, giving the boundaries of the regions in the parameter space where the instability sets in. Figure 3 represents these limiting curves for $a / M=0.2$ and different values of $l_{0}$ and $m$. Clearly, the unstable regions for partial modes with $l_{0}=m=0$ encompass those for higher multipoles.

In Fig. 4 , the boundaries $\left(\omega=0, l_{0}=0\right)$ of the unstable regions are plotted for different values of $a / M$. Fig. 5 highlights a range of $M / \mathrm{R}$ which is not clearly seen in Fig. 4. There, we plot the difference $\xi-\xi^{(0)}$ as a function of

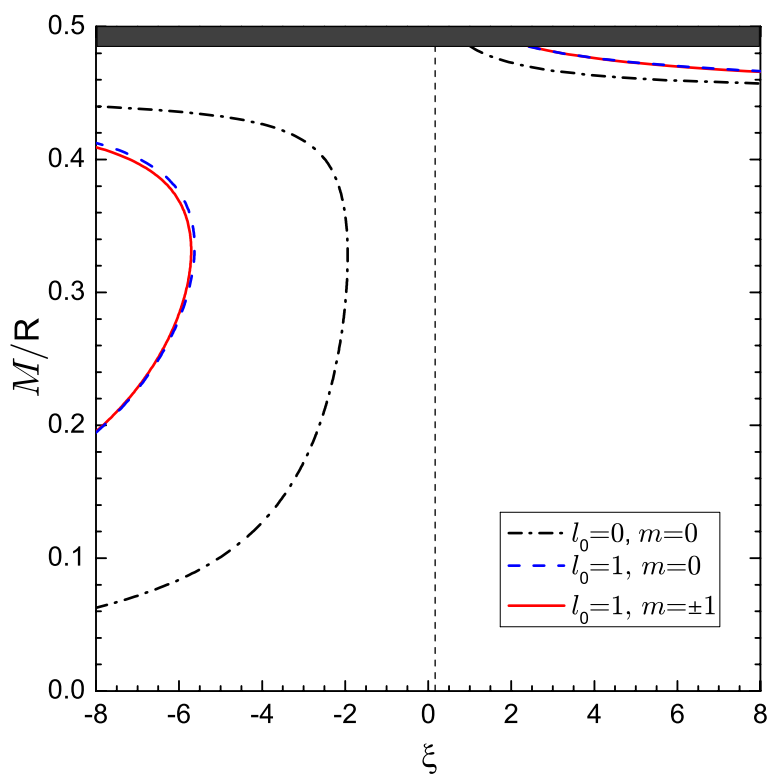

FIG. 3 (color online). Curves characterizing the onset of instability for partial modes with $l_{0}=0$ and $l_{0}=1 \quad(m=0$ and $m= \pm 1$ ). The rotation is fixed to $a / M=0.2$. Curves for the same $l_{0}$ and opposite values of $m$ are degenerate. The plot is restricted to the range of $M / \mathrm{R}$ in which the criterion (41) is valid. The vertical dashed line indicates the conformal-coupling value $\xi=1 / 6$.

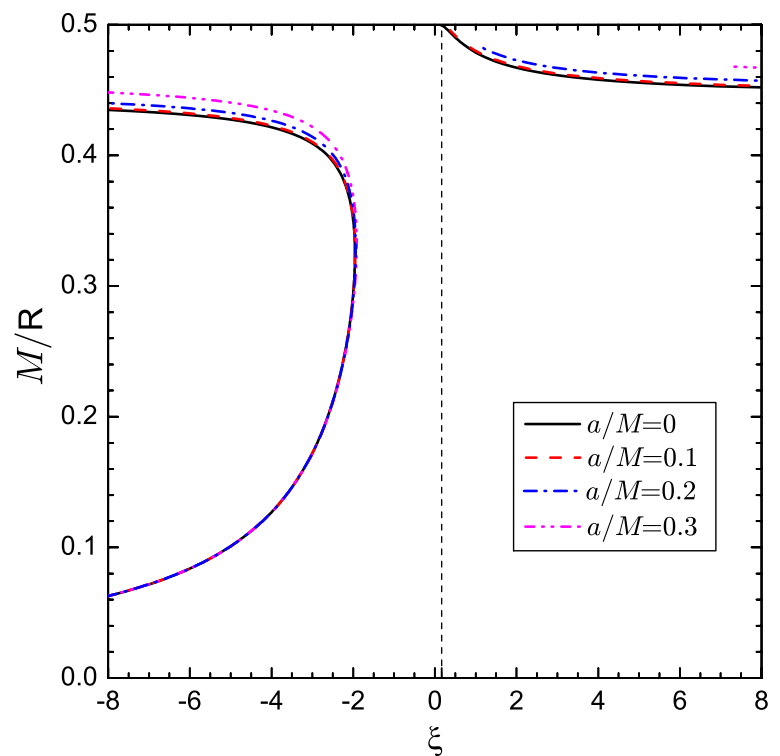

FIG. 4 (color online). Diagram showing the boundaries $(\omega=0$, $\left.l_{0}=0\right)$ of the regions in the parameter space $(\xi, M / \mathrm{R})$ in which the instability is triggered for $a / M=0.1,0.2$, and 0.3 . The static $a=0$ case is plotted for comparison. The vertical dashed line indicates the conformal-coupling value $\xi=1 / 6$. Configurations allowing for tachyonic modes are those to the left of the curves on the left-hand side and to the right of those on the right-hand side. The curves are restricted to the range of $M / \mathrm{R}$ in which the criterion (41) is valid.

$M / \mathrm{R}$, where $\xi^{(0)}$ and $\xi$ are given in Eqs. (63) and Eq. (67), respectively. From Figs. 4 and 5, we conclude that rotation shifts these boundaries to the right, so that the unstable region for negative values of $\xi$ gets enlarged and the one for

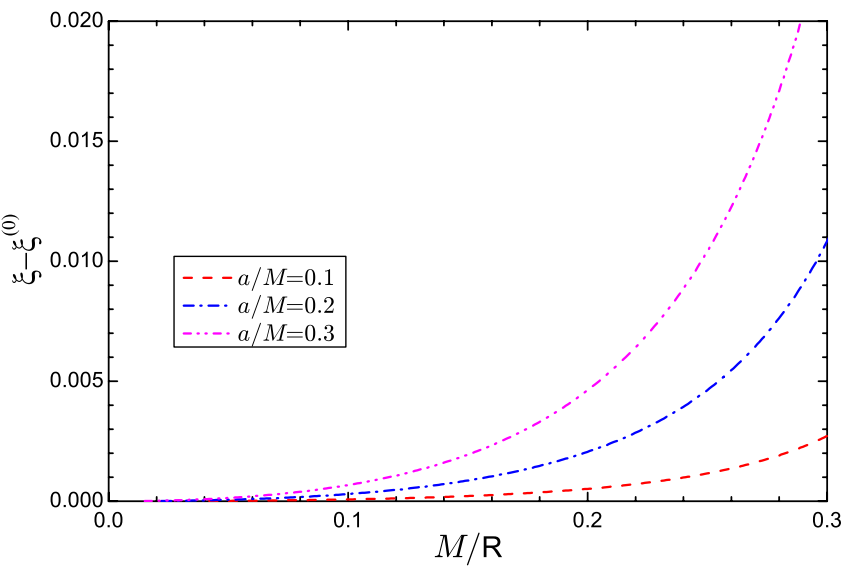

FIG. 5 (color online). Difference $\xi-\xi^{(0)}$ between the values of $\xi$ describing the borders of the unstable regions for a rotating shell and for a static configuration with the same mass-to-radius ratio, as a function of $M /$ R. Here, $l_{0}=m=0$ and $a / M=0.1,0.2$, and 0.3 . We see from the plot that for these values of $a / M$, $\left(\xi-\xi_{0}\right) / \xi_{0} \lesssim(a / M)^{4}$, which suggests that for $0 \leq M / R \lesssim 0.3$, a higher-order analysis would be necessary to extract reliable conclusions. 


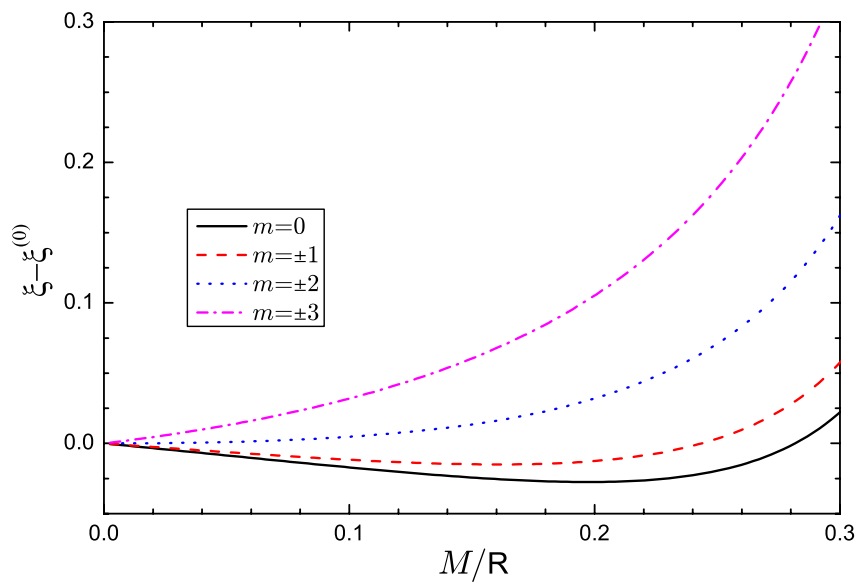

FIG. 6 (color online). Difference $\xi-\xi^{(0)}$ between the values of $\xi$ describing the borders of the unstable regions for a rotating shell and for a static configuration with the same mass-to-radius ratio, as a function of $M / \mathrm{R}$. Here, $a / M=0.3, l_{0}=3$ and $m=0, \pm 1, \pm 2$, and \pm 3 .

positive values of $\xi$ is diminished. The absolute effect, however, turns out to be relatively small (as expected, since this is a second-order correction).

Finally, Fig. 6 shows the value of $\xi-\xi^{(0)}$ as a function of $M / \mathrm{R}$ for $a / M=0.3, l_{0}=3$, and $m=0, \pm 1, \pm 2, \pm 3$. Unlike the $l_{0}=0$ case, it turns out that for higher multipoles $\xi-\xi^{(0)}$ is not everywhere positive. Here and in Fig. 3 it is clear that a reversal in the direction of rotation (achieved either by $a \rightarrow-a$ or by $m \rightarrow-m$ ) has no effect in the parameter space of the instability, which is a direct consequence of the general result of Sec. III. There is nonetheless a coupling between the object rotation and the field angular momentum in higher orders [manifested, e.g., by the term $a^{2} m^{2}$ in Eq. (48)], which breaks the degeneracy in $m$ that is characteristic of the static limit.

Before concluding, it is worthwhile to make a brief comment on the relation between our results concerning the linear instability of nonminimally coupled fields in the spacetime of rotating bodies and the nonlinear effect known as spontaneous scalarization, which was established in the context of scalar-tensor theories in Ref. [24] (see also Refs. [25-27]). In Ref. [7], it was argued that the boundaries of the regions in parameter space which characterize the type of linear instability considered here also delimit the regions where spontaneous scalarization can occur. Although the argument was made in a context of spherical symmetry, the same reasoning seems to apply to our stationary spacetime. Indeed our conclusions are in agreement with a recent result [28], which numerically showed that scalarized rapidly rotating neutron stars exist for a larger range of (negative) couplings than in the static case (see Figs. 4 and 5).

\section{CONCLUSIONS}

Nonminimally coupled free scalar fields are unstable in the spacetime of compact objects for a wide range of field couplings and compact object parameters. Such an instability will be unavoidably triggered by vacuum fluctuations (see Sec. II). This "awakening" of the quantum vacuum was previously treated in the context of spacetimes which were static in the asymptotic past and future associated with the formation of a nonrotating compact object from initially diluted matter. Here, we have investigated how the instability is influenced when the compact object acquires some rotation. In order to also allow a quantum mechanical treatment of the instability, we have discussed the canonical quantization of the scalar field in a spacetime which is nearly flat in the asymptotic past and stationary and axisymmetric in the future. As a prototype model for our compact spinning object, we have considered the spacetime of a spinning thin shell. As explained in Sec. III, in order to obtain nontrivial results concerning the role of rotation on the instability parameter space we had to go beyond first order in the object angular momentum (see also Sec. V). The simple thin shell model is justified, thus, since it allowed us to push the analytical treatment further. Our main result is expressed in Eq. (67) and depicted in Figs. 2-6. In particular, we observe that the regions in parameter space which characterize the instability of a partial mode with a certain value of $l_{0}$ are invariant under $m \rightarrow-m$ but are nondegenerate in $m$ as can be seen in Figs. 3 and 6 (in contrast to the static case). Figure 3 also shows that the instability first sets in by partial modes with $l_{0}=0$. Our analysis suggests that the overall effect of (slow) rotation is to enlarge the instability parameter space for negative values of $\xi$ and to diminish the one for positive values of $\xi$ (see Figs. 4 and 5), in agreement with recent results in the context of scalar-tensor theories [28].

\section{ACKNOWLEDGMENTS}

R. M. was supported by the São Paulo Research Foundation (FAPESP) under Grant No. 2011/06429-3. G. M. and D. V. acknowledge partial support from FAPESP under Grants No. 2007/55449-1 and No. 2013/ 12165-4, respectively. G. M. also acknowledges Conselho Nacional de Desenvolvimento Científico e Tecnológico (CNPq) for partial support. 
[1] W. C. C. Lima and D. A. T. Vanzella, Phys. Rev. Lett. 104, 161102 (2010).

[2] W. C. C. Lima, G. E. A. Matsas, and D. A. T. Vanzella, Phys. Rev. Lett. 105, 151102 (2010).

[3] A. G. S. Landulfo, W. C. C. Lima, G. E. A. Matsas, and D. A. T. Vanzella, Phys. Rev. D 86, 104025 (2012).

[4] R. F. P. Mendes, G. E. A. Matsas, and D. A. T. Vanzella, Phys. Rev. D 89, 047503 (2014).

[5] T. Harada, Prog. Theor. Phys. 98, 359 (1997); Phys. Rev. D 57, 4802 (1998).

[6] V. Cardoso, I. P. Carucci, P. Pani, and T. P. Sotiriou, Phys. Rev. Lett. 111, 111101 (2013).

[7] P. Pani, V. Cardoso, E. Berti, J. Read, and M. Salgado, Phys. Rev. D 83, 081501 (2011).

[8] W. C. C. Lima, R. F. P. Mendes, G. E. A. Matsas, and D. A. T. Vanzella, Phys. Rev. D 87, 104039 (2013).

[9] J. W. Hessels, S. M. Ransom, I. H. Stairs, P. C. C. Freire, V. M. Kaspi, and F. Camilo, Science 311, 1901 (2006). See also the Australia Telescope National Facility pulsar database, www.atnf.csiro.au/research/pulsar/psrcat/.

[10] J. L. Friedman and N. Stergioulas, Rotating Relativistic Stars (Cambridge University Press, Cambridge, England, 2013).

[11] W. G. Unruh, Phys. Rev. D 10, 3194 (1974).

[12] L. H. Ford, Phys. Rev. D 12, 2963 (1975).

[13] A. L. Matacz, P. C. W. Davies, and A. C. Ottewill, Phys. Rev. D 47, 1557 (1993).

[14] W. C. C. Lima, Phys. Rev. D 88, 124005 (2013); B. Schroer and J. A. Swieca, ibid. 2, 2938 (1970); B. Schroer, ibid. 3, 1764 (1971).
[15] S. A. Fulling, Aspects of Quantum Fields Theory in Curved Spacetime (Cambridge University Press, Cambridge, England, 1989).

[16] N. D. Birrell and P.C.W. Davies, Quantum Fields in Curved Space (Cambridge University Press, Cambridge, England, 1982).

[17] C. Kuo and L. H. Ford, Phys. Rev. D 47, 4510 (1993).

[18] V. de la Cruz and W. Israel, Phys. Rev. 170, 1187 (1968).

[19] E. Poisson, A Relativist's Toolkit (Cambridge University Press, Cambridge, England, 2004)

[20] C. Flammer, Spheroidal Wave Functions (Stanford University Press, Stanford, 1957).

[21] J. L. Friedman and B. F. Shutz, Astrophys. J. 200, 204 (1975).

[22] In our figures higher-order contributions will be also present but a number of numerical tests leads us to believe that they will not change our physical conclusions.

[23] S. Hassani, Mathematical Physics: A Modern Introduction to Its Foundations (Springer, New York, 1999).

[24] T. Damour and G. Esposito-Farese, Phys. Rev. Lett. 70, 2220 (1993).

[25] J. Novak, Phys. Rev. D 57, 4789 (1998); 58, 064019 (1998).

[26] M. Salgado, D. Sudarsky, and U. Nucamendi, Phys. Rev. D 58, 124003 (1998).

[27] H. Sotani, Phys. Rev. D 86, 124036 (2012).

[28] D. D. Doneva, S. S. Yazadjiev, N. Stergioulas, and K. D. Kokkotas, Phys. Rev. D 88, 084060 (2013). 